\title{
COSMICITY OF COMETRIZABLE SPACES
}

\author{
GARY GRUENHAGE
}

\begin{abstract}
A space $X$ is cometrizable if $X$ has a coarser metric topology such that each point of $X$ has a neighborhood base of metric closed sets. Most examples in the literature of spaces obtained by modifying the topology of the plane or some other metric space are cometrizable. Assuming the Proper Forcing Axiom (PFA) we show that the following statements are equivalent for a cometrizable space $X:$ (a) $X$ is the continuous image of a separable metric space; (b) $X^{\omega}$ is hereditarily separable and hereditarily Lindelöf, (c) $X^{2}$ has no uncountable discrete subspaces; (d) $X$ is a Lindelöf semimetric space; (e) $X$ has the pointed ccc. This result is a corollary to our main result which states that, under PFA, if $X$ is a cometrizable space with no uncountable discrete subspaces, then either $X$ is the continuous image of a separable metric space or $X$ contains a copy of an uncountable subspace of the Sorgenfrey line.
\end{abstract}

\section{INTRODUCTION}

A space ${ }^{1} X$ is cosmic if it is the continuous image of a separable metric space, or equivalently, if it has a countable network (i.e., a countable collection $\mathscr{N}$ such that whenever $U$ is a neighborhood of a point $x \in X$, then $x \in N \subset U$ for some $N \in \mathscr{N}$ ).

Several authors have asked whether a space $X$ must be cosmic if $X$ satisfies some specific conditions; for example, is $X$ cosmic if

(1) $X^{\omega}$ is hereditarily separable and hereditarily Lindelö?

(2) $X$ is a Lindelöf semimetric ${ }^{2}$ space?

(3) $X$ has the pointed ccc?

Question (2) is due to R. W. Heath [H]. E. Michael [M] used CH to construct a counterexample which is a subset of the plane with the "bow-tie" topology. Other examples have been constructed under $\mathrm{CH}$ by E. S. Berney [Br] and N. V. Veličko [V]. Michael's example is also a counterexample to question (1), which is due to A. V. Arhangel' skii [A]. Michael also showed that under $\mathrm{CH}$ there is

Received by the editors January 22, 1987 and, in revised form, December 20, 1987.

1980 Mathematics Subject Classification (1985 Revision). Primary 54E20, 54A35.

Partially supported by an NSF grant.

${ }^{1}$ All spaces are regular and $T_{1}$.

$2 X$ is a semimetric space if one can assign to each $x \in X$ a neighborhood base $\{b(x, n)\}_{n \in \omega}$ such that $y \in b(x, n) \Leftrightarrow x \in b(y, n)$. It is easy to check that this is equivalent to the usual definition which says that there is a "metric" $d$ on $X$ that satisfies all the standard conditions except for the triangle inequality such that $\varepsilon$-balls about $x \in X$ form a neighborhood base at $x$. 
a subspace of the Sorgenfrey line which is a counterexample to (1). Question (3) is due to Tkačenko [Tk]. Recall that $X$ has the pointed ccc if, whenever $\left\{x_{\alpha}\right\}_{\alpha<\omega_{1}} \subset X$ and $U_{\alpha}$ is a neighborhood of $x_{\alpha}$ for each $\alpha$, there exists $\beta \neq \alpha$ with $x_{\beta} \in U_{\alpha}$ and $x_{\alpha} \in U_{\beta}$. It is easy to see that Lindelöf semimetric spaces have the pointed ccc; thus Michael's example is a counterexample to (3) also. Recently, K. Ciesielski [C] showed that there is a counterexample to (1) and (3) which is consistent with Martin's Axiom (MA) plus $2^{\aleph_{0}}=\aleph_{2}$. No ZFC counterexamples are known.

A space $X$ is cometrizable if there is a weaker metrizable topology on $X$ such that each point of $X$ has a (not necessarily open) neighborhood base consisting of sets which are closed in the metric topology. For example, the $\mathrm{CH}$ examples mentioned above cometrizable, as are most of the examples constructed in the 50 's and 60's by modifying the topology of the plane or some other metric space. Other examples worth mentioning are the density topology [Ta], the Kunen line [JKR], and van Douwen and Kunen's $S$ - and $L$-subspaces of $P(\omega)$ [vDK].

The purpose of this note is to show that the Proper Forcing Axiom (PFA) implies positive answers to all three questions for the class of cometrizable spaces. PFA is a strengthening of $\mathrm{MA}_{\omega_{1}}$ which asserts that, given $\aleph_{1}$ dense subsets of a "proper" poset (partially ordered set) $P$, there is some filter contained in $P$ which meets them all. We shall not need to know the definition of "proper", but only that countably closed posets and ccc posets are proper, and finite iterations of proper posets are proper. For more about PFA, see Baumgartner $\left[\mathrm{B}_{1}\right]$ or Shelah [S].

Main Result. (PFA) Let $X$ be a cometrizable space with no uncountable discrete subspace. Then either

(a) $X$ contains a copy of an uncountable subspace of the Sorgenfrey line; or

(b) $X$ is cosmic.

One easily obtains positive answers for cometrizable spaces to questions (2) and (3) from this, because no uncountable subspace of the Sorgenfrey line has the pointed ccc, and every Lindelöf semimetric space has the pointed ccc. To get (1), observe that Baumgartner's result that, under PFA, all $\aleph_{1}$-dense subsets of the reals are order isomorphic (see $\left[\mathbf{B}_{2}\right.$ or $\left.\mathbf{B}_{1}\right]$ ) implies that the square of an uncountable subset $S$ of the Sorgenfrey line contains an uncountable discrete set (because we may assume that $x \in S$ implies $-x \in S$, so $S^{2} \supset$ $\{(x,-x): x \in S\})$. Hence the statement " $X^{2}$ has no uncountable discrete subspace" is sufficient, under PFA, to imply that a cometrizable space $X$ is cosmic.

Martin's Axiom (MA) is not sufficient to obtain the main result (or the aforementioned consequences). This is shown for general spaces by Ciesielski's example. His example has the pointed ccc, hence has no uncountable discrete or Sorgenfrey subspaces, and is not cosmic. However, this example does not appear to be cometrizable. We can obtain a cometrizable counterexample to (1) 
consistent with $\mathrm{MA}_{\omega_{1}}$ using the notion of an "increasing set" due to Abraham, and Shelah [AS].

Definition. An uncountable subset $A$ of the real line is increasing if whenever $n \in \omega$ and $\left\{\vec{a}_{\alpha}=\left(a_{\alpha i}\right)_{i<n}: \alpha<\omega_{1}\right\}$ is a collection of pairwise disjoint (as unordered sets) $n$-tuples from $A$, there exists $\beta \neq \alpha$ such that $a_{\beta i}<a_{\alpha i}$ for all $i<n$.

It is shown in [AS] that " $\mathrm{MA}_{\omega_{1}}+\exists$ an increasing set" is consistent with ZFC; another proof is given in [ARS].

Now observe that if $A \subset R$ is increasing, and $A$ is given the right halfopen interval topology, then $A^{\omega}$ is hereditarily separable and hereditarily Lindelöf, ${ }^{3}$ so we have a cometrizable counterexample to Arhangel' skii's question consistent with $\mathrm{MA}_{\omega_{1}}$. To prove this, it suffices to prove that $A^{n}$ is hereditarily separable and hereditarily Lindelöf for each $n \in \omega$, and the definition of "increasing" seems almost tailor-made for showing this-so we leave it as an exercise for the reader.

To get the cometrizable counterexample to the main result (and questions (2) and (3) on cosmicity as well) consistent with $\mathrm{MA}_{\omega_{1}}$, we use the notion of an "2-entangled" set.

Definition. An uncountable subset $A$ of $R$ is 2-entangled if there is no uncountable monotone function $f$ from a subset of $A$ to $A$ with no fixed points.

Shelah showed in [AS] that the existence of a 2-entangled set is consistent with $\mathrm{MA}_{\omega_{1}}$.

Now to get our example, let $\vec{A}=\left\{\vec{a}_{\alpha}=\left(a_{\alpha 0}, a_{\alpha 1}\right): \alpha<\omega_{1}\right\}$ be a collection of ordered pairs from a 2-entangled set $A$ such that $a_{\alpha i}=a_{\beta j}$ implies $\alpha=\beta$ and $i=j$. For $\vec{a} \in A$, let $\vec{x} \in b(\vec{a}, n)$ if and only if the Euclidean distance from $\vec{x}$ to $\vec{a}$ is less than $1 / 2^{n}$, and $\vec{x}$ is either above and to the right of $\vec{a}$, or below and to the left of $\vec{a}$. Then it is easy to check that $\vec{A}$ with this topology generated by the $b(\vec{a}, n)$ 's is a semimetric space, and $\vec{A}$ is hereditarily separable and hereditarily Lindelöf. It follows that $\vec{A}$ has the pointed ccc and does not contain a copy of an uncountable subspace of the Sorgenfrey line. To see that $\vec{A}$ is not cosmic, we use Michael's trick. Let $\vec{A}^{\perp}$ be a topology on $\vec{A}$ defined the same way as above, but with the words "left" and "right" interchanged in the definition of $b(\vec{a}, n)$. Then $\vec{A}^{\perp}$ is also hereditarily Lindelöf, but the "diagonal" in $\vec{A} \times \vec{A}^{\perp}$ is an uncountable discrete space. It is easy to check that the product of a cosmic space and a hereditarily Lindelöf space is hereditarily Lindelöf-hence $\vec{A}$ is not cosmic.

${ }^{3} \mathrm{~S}$. Todorcevic announced this fact at the topology conference in Eger, Hungary, 1983. He also noticed independently the other examples we give here consistent with $\mathbf{M A}_{\omega_{1}}$, and recently he has shown $\left[\mathrm{TO}_{3}\right]$ that the existence of a counterexample to the Main Result is implied by $\mathrm{MA}+2^{\omega} \neq \omega_{2}$. 
The above construction also provides a negative solution to a problem of Juhasz. ${ }^{4} \mathrm{He}$ asks (see $[\mathrm{J}]$ ) if $\mathrm{MA}_{\omega_{1}}$ implies that the pointed ccc property is finitely productive-it is well known that this is true for the ccc property. Let $\vec{A}$ and $\vec{A}^{\perp}$ be disjoint copies of the spaces defined in the preceding paragraph, and let $X$ be their free union. Then $X$ has the pointed ccc, but $X^{2}$ contains an uncountable discrete space, so does not have the pointed ccc. The question remains whether or not PFA (or maybe Martin's Maximum) implies that the pointed ccc is finitely productive.

We do not know if the main result is valid without the "cometrizable" assumption. This question is related to the question of the consistency of the following statement, which is also discussed in [G]:

(*) Every uncountable first countable space contains an uncountable metrizable subspace or a copy of an uncountable subspace of the Sorgenfrey line.

Since no counterexample to $(*)$ can contain an uncountable discrete space, this is really a question about hereditarily ccc spaces. It is easy to show that under $\mathbf{M A}_{\omega_{1}}$, every uncountable cosmic space contains an uncountable metrizable subspace (see §3). Hence our main result shows that $(*)$ holds for cometrizable spaces, even without the first-countable assumption. Indeed, we do not know any counterexample to $(*)$ even without assuming first-countability. We hasten to point out, however, that $S$ - and $L$-spaces yield counterexamples to (*) (consider right/left separated subspaces), so a proof of the consistency of $(*)$ without first-countability would establish the consistency of no $L$-spaces, which is unknown. Another reason for leaving "first-countable" in is that, as shown in [G], a proof of the consistency of $(*)$ as stated would suffice to solve some problems on perfectly normal compacta, which is what got us interested in $(*)$ to begin with. Note that with the first-countability assumption in, PFA implies that any counterexample to $(*)$ is both hereditarily separable and hereditarily Lindelöf, since PFA implies there are no $S$-spaces $\left[\mathrm{To}_{1}\right]$, and $\mathrm{MA}_{\omega_{1}}$ implies there are no first-countable $L$-spaces [Sz].

In $\S 3$, we make some further remarks concerning $(*)$, including a proof that, under $\mathbf{M A}_{\omega_{1}}$, if there is a counterexample to $(*)$, then there is a submetrizable one, i.e., one with a weaker metric topology. This indicates that if $(*)$ fails in general, then perhaps the cometrizable result is best possible.

The author wishes to thank Stewart Baldwin and Robert Beaudoin for valuable discussions concerning this material.

\section{Cosmicity}

The purpose of this section is to prove the main result as stated in the introduction. First we establish some preliminary results.

Lemma 2.1. $\left(\mathrm{MA}_{\omega_{1}}\right)$ Suppose $X \subset R,|X|=\aleph_{1}$, and the topology $\tau$ on $X$ is finer than the Sorgenfrey topology. Suppose also that there is a countable

\footnotetext{
${ }^{4}$ Todorcevic presented a $T_{2}$ counterexample at the ' 83 conference in Eger, Hungary.
} 
collection $\mathscr{F}$ of closed subsets of $X$ such that, for each $x \in X$, the set

$$
\mathscr{F}_{x}=\{F \cap[x, \rightarrow): x \in F \in \mathscr{F}\}
$$

is a net at $x$ (i.e., every neighborhood of $x$ contains a member of $\mathscr{F}_{x}$ ).

Then there is an uncountable subset $Y$ of $X$ such that the topology $\tau$ on $Y$ is the same as the Sorgenfrey topology on $Y$.

Proof. Let $\sigma$ be the separable metric topology on $X$ generated by

$$
\mathscr{E} \cup \mathscr{F} \cup\{X \backslash F: F \in \mathscr{F}\} \text {, }
$$

where $\mathscr{E}$ is the Euclidean topology. Let $f:(X, \mathscr{E}) \rightarrow(X, \sigma)$ be the identity on $X$. By $\left[\mathrm{Fr}_{1}, 23 M(\mathrm{f})\right]$, there is an uncountable $Y \subset X$ such that $f \mid Y$ is continuous, hence a homeomorphism from $(Y, \mathscr{E} \mid Y)$ to $(Y, \sigma \mid Y)$. Then $y \in Y$ and $F \in \mathscr{F}_{y}$ implies $F \supset[y, y+\varepsilon) \cap Y$ for some $\varepsilon>0$, so $\tau$ is the same as the Sorgenfrey topology on $Y$.

The following lemma is due to $\mathrm{D}$. H. Fremlin $\left[\mathrm{Fr}_{2}\right]$. For the sake of completeness, we give his proof.

Lemma $2.2\left[\mathrm{Fr}_{2}\right]$. Let $X$ be a second countable space, and suppose $U \subset X^{2}$ has open vertical (or horizontal) cross-sections. Then there is a finer second-countable topology on $X$ with respect to which $U$ is open.

Proof. If $\mathscr{B}$ is a countable base for a topology with respect to which $U$ has open vertical cross-sections, then the topology generated by $\mathscr{B}$ together with the sets $\hat{B}=\{x: B \subset\{y:(x, y) \in U\}\}$ for $B \in \mathscr{B}$ is the desired finer secondcountable topology.

Lemma 2.3. (CH) Suppose $X \subset R$ is uncountable, and for each $x \in X$, we have assigned a set $C_{x} \subset[x, \rightarrow)$ which is closed in the Euclidean topology. Suppose also that there does not exist an uncountable $Y \subset X$ satisfying:

(a) $y<y^{\prime} \in Y \Rightarrow y^{\prime} \in C_{y}$.

Then there is a ccc poset $Q$ such that in $V^{Q}$, there is an uncountable $Z \subset X$ satisfying:

(b) $z<z^{\prime} \in Z \Rightarrow z^{\prime} \notin C_{z}$.

Remark. Originally, we had a direct proof of Lemma 2.3. But Fremlin pointed out to the author that Lemma 2.2 can be used to show that this lemma follows form a similar lemma of Abraham, Rubin and Shelah which is used to prove the consistency of their "semi-open coloring axiom (SOCA)".

SOCA. ${ }^{5}$ Let $X$ be an uncountable second-countable space, and let $U$ be a symmetric open subset of $X^{2} \backslash \Delta$, where $\Delta$ is the diagonal. Then there is an uncountable $Y \subset X$ such that either $Y^{2} \cap U=\varnothing$ or $Y^{2} \backslash \Delta \subset U$.

${ }^{5} \mathrm{D}$. H. Fremlin $\left[\mathrm{Fr}_{2}\right]$ observed that the arguments of this paper can be reworked to show that our Main Result is implied by a stronger version of SOCA in which the conclusion is that either $X=\bigcup_{n \in \omega} Y_{n}$ such that $Y^{2} \cap U=\varnothing$, or there is an uncountable $Y \subset X$ with $Y^{2} \backslash \Delta \subset U$. This version of SOCA follows from PFA using arguments in [ARS]. (Except that the size of $X$ is unrestricted, it is the same as SOCAl in [ARS].) 
The lemma from which the consistency of SOCA follows by standard arguments is:

Lemma 2.4 [ARS]. (CH) Let $X$ be a second countable space, and let $U$ be a symmetric open subset of $X^{2} \backslash \Delta$. If there does not exist an uncountable $Y \subset X$ such that $Y^{2} \cap U=\varnothing$, then there is a ccc poset $Q$ of size $\aleph_{1}$ such that in $V^{Q}$, there is an uncountable $Z \subset X$ with $Z^{2} \backslash \Delta \subset U$.

Proof of Lemma 2.3. Let $X$ and $\left\{C_{x}: x \in X\right\}$ be as hypothesized. Define $U \subset X^{2} \backslash \Delta$ by putting $(x, y) \in U$ if and only if $x<y$ and $y \notin C_{x}$, or $y<x$ and $x \notin C_{y}$. The hypotheses of Lemma 2.3 imply that there is no uncountable $Y \subset X$ with $Y^{2} \cap U=\varnothing$. Now $U$ is the union of a set with open vertical cross-sections and a set with open horizontal cross-sections. By Lemma 2.2, $U$ is open in some finer second countable topology. Thus Lemma 2.4 applies, and Lemma 2.3 follows.

Lemma 2.5. ${ }^{6}$ (SOCA) There do not exist cometrizable S-or L-spaces.

Proof. Suppose $X$ is a cometrizable $S$-space. Then there is an uncountable left-separated subspace $\left\{x_{\alpha}: \alpha<\omega_{1}\right\}$ (i.e., $x_{\alpha} \notin \overline{\left\{x_{\beta}: \beta<\alpha\right\}}$ for each $\alpha<$ $\left.\omega_{1}\right)$. By passing to a subspace if necessary, we may assume $X \subset R$ and $X$ is cometrizable with respect to the Euclidean topology. For each $\alpha<\omega_{1}$, choose a Euclidean closed $C_{x_{u}}$ containing $x_{\alpha}$ such that $C_{x_{u}} \cap\left\{x_{\beta}: \beta<\alpha\right\}=\varnothing$. Now a double application of SOCA in the same way that Lemma 2.4 was applied to obtain Lemma 2.3 shows that there exists an uncountable $Y \subset\left\{x_{\alpha}: \alpha<\omega_{1}\right\}$ such that for $y \neq y^{\prime} \in Y$, whether $y \in C_{y}^{\prime}$ or not depends only on whether $y<y^{\prime}$ or not. But this is easily seen to be impossible. Thus there are no cometrizable $S$-spaces; the proof that there are no cometrizable $L$-spaces is similar.

Much of the work of the proof of the main result is done in the following proposition:

Proposition 2.6. (PFA) Let $X$ be an uncountable cometrizable space. Then $X$ contains either an uncountable metrizable subspace or a copy of an uncountable subspace of the Sorgenfrey line.

Proof. Suppose $(X, \tau)$ is a counterexample. Then $X$ has no uncountable discrete subspaces, hence any weaker metric topology is separable. So by passing to a subspace if necessary, we may assume $X \subset R$ and $X$ is cometrizable with respect to the Euclidean topology.

${ }^{6}$ For the proof of the Main Result, one could just as well use the fact that there are no cometrizable $S$ - or $L$-spaces under $\mathrm{MA}_{\omega_{1}}$. This fact was noticed independently by $\mathrm{J}$. Chaber and $\mathrm{S}$. Todorcevic, the latter of whom pointed out that this follows directly from Lemma 13 in $\left[\mathrm{To}_{2}\right]$. 
Let $\tau^{+}$(resp., $\tau^{-}$) be the topology on $X$ generated by $\tau$ together with the right (resp., left) half-open interval topology. Since no uncountable subspace of $X$ is metrizable or Sorgenfrey, either

(i) no uncountable subspace of $\left(X, \tau^{+}\right)$is Sorgenfrey or discrete; or

(ii) there exists an uncountable $X^{\prime} \subset X$ such that no uncountable subspace of $\left(X^{\prime}, \tau^{-}\right)$is (left) Sorgenfrey or discrete.

Without loss of generality, we assume (i).

Let $P$ be a countably closed poset collapsing the continuum $\underline{c}$ to $\omega_{1}$; then $V^{P} \Vdash \mathrm{CH}$. Now our plan is to use $\mathrm{CH}$ in $V^{P}$ to set up an application of Lemma 2.3 , obtaining a ccc poset $Q$ such that, in $V^{P * Q},\left(X, \tau^{+}\right)$contains an uncountable discrete subspace. Once we have done this, PFA implies that there exists in $V$ an uncountable discrete subspace of $\left(X, \tau^{+}\right)$, because $P * Q$ is proper and only $\aleph_{1}$-many dense sets are needed to determine a discrete set of size $\aleph_{1}$ and the open neighborhoods which witness discreteness. But this contradicts (i) above.

By Lemma 2.5 , since $\left(X, \tau^{+}\right)$has no uncountable discrete subspaces, $\left(X, \tau^{+}\right)$ is hereditarily separable and hereditarily Lindelöf in $V$. We need to know that $\left(X, \tau^{+}\right)$remains hereditarily separable and hereditarily Lindelöf in $V^{P}$. This is done by a standard argument for countably closed forcings. If, for example, $\left(X, \tau^{+}\right)$is not hereditarily separable in $V^{P}$, then it contains an uncountable left-separated subspace $Y=\left\{y_{\alpha}: \alpha<\omega_{1}\right\}$. Let $\mathbf{Y}$ be a name for $Y$. We can inductively construct (in $V$ ) sequences $\left\{x_{\alpha}: \alpha<\omega_{1}\right\} \subset X$ and $\left\{p_{\alpha}: \alpha<\omega_{1}\right\} \subset$ $P$ such that $p_{\alpha} \leq p_{\beta}$ for $\beta<\alpha$, and $p_{\alpha} \Vdash \check{x}_{\alpha} \in \mathbf{Y}$ and $\check{x}_{\alpha} \notin\left\{\overline{\check{x}_{\beta}: \beta<\alpha}\right\}$. Since a basis for $\left(X, \tau^{+}\right)$in $V$ is also a basis in $V^{P}$, we see that $\left\{x_{\alpha}: \alpha<\omega_{1}\right\}$ must be a left-separated subspace of $X$ in $V$, and we have a contradiction.

Since $P$ is countably closed, any $\omega$-sequence of elements of $V$ in $V^{P}$ is in fact in $V$. Thus in $V^{P}$, there is no countable collection $\mathscr{F}$ satisfying the conditions of Lemma 2.1: otherwise, each $F \in \mathscr{F}$, being the complement of a countable union of members of a base for $\tau^{+}$, would be in $V$, so $\mathscr{F}$ itself would be in $V$.

Still in $V^{P}$, let $\left\{Z_{\alpha}: \alpha<\omega_{1}\right\}$ enumerate all countable subsets of $X$. Inductively choose $x_{\alpha} \in X$ and a Euclidean closed neighborhood $C_{x_{n}} \subset\left[x_{\alpha}, \rightarrow\right)$ of $x_{\alpha}$ such that:

$$
\beta<\alpha \Rightarrow\left(x_{\alpha} \notin \bar{Z}_{\beta} \text { or } \bar{Z}_{\beta} \cap\left[x_{\alpha}, \rightarrow\right) \subseteq C_{x_{n}}\right) .
$$

This can be done because $\left\{\bar{Z}_{\beta}: \beta<\alpha\right\}$ cannot satisfy the conditions of the collection $\mathscr{F}$ of Lemma 2.1 .

Let $X^{\prime}=\left\{x_{\alpha}: \alpha<\omega_{1}\right\}$. We show that $\left\{C_{x}: x \in X^{\prime}\right\}$ satisfies the conditions of Lemma 2.3. Suppose $Y \subset X$ is uncountable, and $y<y^{\prime} \in Y$ implies $y^{\prime} \in C_{y}$. For some $\beta<\omega_{1}, Z_{\beta}$ is a dense subset of $Y$. Choose $x_{\alpha} \in Y$ with $\alpha>\beta$. Then $x_{\alpha} \in \bar{Z}_{\beta}$, and $Z_{\beta} \cap\left[x_{\alpha}, \rightarrow\right) \subset C_{x_{i}}$, so $\bar{Z}_{\beta} \cap\left[x_{\alpha}, \rightarrow\right) \subset C_{x_{0}}$, which contradicts the way the $x_{\alpha}$ 's and $C_{x_{n}}$ 's were chosen. 
Thus there is a ccc poset $Q$ such that in $V^{P * Q}$ there is an uncountable $Z \subset X^{\prime}$ such that $z<z^{\prime} \in Z$ implies $z^{\prime} \in C_{z}$. This means $\left(Z, \tau^{+}\right)$is discrete. So PFA implies that in $V,\left(X, \tau^{+}\right)$contains an uncountable discrete subset (as we discussed earlier), which is a contradiction.

Proof of Main Result. Suppose $X$ is cometrizable, with no uncountable discrete subspace and no countable network. Our aim is to show that $X$ has an uncountable weakly separated subset $Z$; i.e., there are neighborhoods $\left\{U_{z}: z \in Z\right\}$ such that, for $z, z^{\prime} \in Z, z \in U_{z}$ and $z \neq z^{\prime}$ implies $z \notin U_{z}$, or $z^{\prime} \notin U_{z}$. Then no uncountable subspace of $Z$ is metrizable, so by Proposition 2.6, $Z$ contains a copy of an uncountable subspace of the Sorgenfrey line.

Let $P$ be a countably closed poset collapsing $\underline{c}$ to $\omega_{1}$. As in the proof of Proposition 2.6, $X$ remains hereditarily separable and hereditarily Lindelöf in $V^{P}$. One also easily shows that $X$ cannot have a countable network in $V^{P}$, or it would in $V$, too.

In $V^{P}$, let $\left\{Z_{\alpha}: \alpha<\omega_{1}\right\}$ index all countable subsets of $X$, and inductively choose $x_{\alpha} \in X$ and a metric-closed neighborhood $C_{x_{\alpha}}$ of $x_{\alpha}$ such that: "there does not exist $\beta<\alpha$ with $x_{\alpha} \in \bar{Z}_{\beta} \subset C_{x_{n}}$ ". (We can do this because $\left\{\bar{Z}_{\beta}: \beta<\right.$ $\alpha\}$ is not a network for $X$.)

Let $X^{\prime}=\left\{x_{\alpha}: \alpha<\omega_{1}\right\}$. By passing to a subspace if necessary, we may assume that $X^{\prime} \subset R$; and each $C_{x} \cap X^{\prime}, x \in X^{\prime}$, is Euclidean closed.

Claim. There is an uncountable $Y \subset X^{\prime}$ such that either

(i) no uncountable $Z \subset Y$ satisfies " $z<z^{\prime} \in Z \Rightarrow z^{\prime} \in C_{z}$ "; or

(ii) no uncountable $Z \subset Y$ satisfies " $z<z^{\prime} \in Z \Rightarrow z \in C_{z^{\prime}}$ ".

To see the claim, if taking $Y=X^{\prime}$ does not work, take an uncountable $Z \subset X^{\prime}$ satisfying the condition of (i), and let $Y=Z$. This $Y$ satisfies (ii), for if $W \subset Y$ satisfies the condition in (ii), then

$$
\text { “ } w \neq w^{\prime} \in W \Rightarrow w \in C_{w^{\prime}},,
$$

so $C_{w} \supset W$ for each $w \in W$. Now some $Z_{\beta}$ is dense in $W$, and if $x_{\alpha} \in W$ with $\alpha>\beta$, then $x_{\alpha} \in \bar{Z}_{\beta} \subset C_{x_{l}}$, a contradiction.

Now by Lemma 2.3 , there is a ccc poset $Q$ such that in $V^{P * Q}$, there is an uncountable $Z \subset Y$ so that either

(iii) $z<z^{\prime} \in Z \Rightarrow z^{\prime} \notin C_{z}$; or

(iv) $z<z^{\prime} \in Z \rightarrow z \notin C_{z^{\prime}}$.

In particular,

$$
\text { “ } z \neq z^{\prime} \in Z \Rightarrow z^{\prime} \notin C_{z} \text { or } z \notin C_{z^{\prime}} " .
$$

Since $P * Q$ is proper and PFA holds, such an uncountable set $Z$ and assignment $\left\{C_{z}: z \in Z\right\}$ exists in $V$. The proof is completed as discussed earlier.

We now show that the main result holds for a somewhat different class of spaces. This class of spaces is more general than the "butterfly spaces" of 
D. Burke and E. van Douwen [BvD]. In their paper they make a statement weaker than

"In ZFC, there is probably no semimetrizable Lindelöf butterfly space which is not cosmic."

The next proposition shows that their hunch was correct.

Proposition 2.7. (PFA) Let $X$ be a space having a weaker metrizable topology such that for each point $x \in X$, the collection $\{U: U \backslash\{x\}$ is metric open $\}$ contains a base at $x$. If $X$ contains no uncountable discrete subspace and no copy of an uncountable subspace of the Sorgenfrey line, then $X$ is cosmic.

Proof. The proof follows in the same way as the proof of the Main Result, using the next lemma in place of Lemma 2.1 .

Lemma 2.8. $\left(\mathrm{MA}_{\omega_{1}}\right)$ Suppose $X \subset R,|X|=\aleph_{1}$, the topology $\tau$ on $X$ is finer than the Sorgenfrey topology, and each point $x \in X$ has a base of sets of the form $U$ such that $U \backslash\{x\}$ is Euclidean open. If there is a countable collection $\mathscr{F}$ of closed subsets of $X$ such that, for each $x \in X$, the Euclidean closure of any given neighborhood of $x$ contains a member of

$$
\{F \cap[x, \rightarrow): x \in F \in \mathscr{F}\},
$$

then there is an uncountable $Y \subset X$ such that the topology $\tau$ on $Y$ is the same as the Sorgenfrey topology on $Y$.

Proof. As in the proof of Lemma 2.1, there is an uncountable $Y \subset X$ such that the Euclidean closure of each neighborhood of $y \in Y$ contains $[y, y+\varepsilon) \cap Y$ for some $\varepsilon>0$. It is not difficult to use $\mathscr{F}$ to show that $X$ is hereditarily separable and hereditarily Lindelöf. Thus we may assume that $Y$ is $\aleph_{1}$-dense (with respect to $\tau$ ).

Using the facts that $(X, \tau)$ contains no uncountable discrete subspaces, and that each $x \in X$ has a base of neighborhoods of the form $U$ with $U \backslash\{x\}$ Euclidean open, it is easy to check that

$$
\left|\mathrm{cl}_{\mathscr{E}}(A) \backslash \mathrm{cl}_{\tau}(A)\right| \leq \aleph_{0}
$$

for any $A \subset X$. It follows that the $\tau$-closure of each neighborhood of $y \in Y$ contains $[y, y+\varepsilon) \cap Y$ for some $\varepsilon>0$, and so by regularity the topology $\tau$ on $Y$ is the same as the Sorgenfrey topology.

As the reader is probably aware, the consistency of PFA implies the consistency of large cardinals, hence PFA cannot be proven consistent from ZFC alone. Thus any result obtained under the assumption of PFA begs the question of whether a large cardinal assumption is really necessary, i.e., can the result be shown consistent assuming only the consistency of ZFC. In a letter to the author, S. Todorcevic showed that large cardinals are not necessary. With his kind permission, we outline his argument here.

The appropriate model is obtained from a model of $\mathrm{CH}$ plus $\diamond_{\omega_{2}}\left\{\delta<\omega_{2}: \operatorname{cf} \delta=\omega_{1}\right\}$, via a finite support iteration $\left\langle P_{\alpha}, \dot{Q}_{\alpha}: \alpha<\omega_{2}\right\rangle$ of 
ccc posets of size $\aleph_{1}$. As we shall see, the diamond principle will allow us to carry out a kind of reflection argument in which supposed counterexamples in the final model get destroyed at some earlier stage.

At successor stages, force with ccc posets that will result in MA and SOCA holding in the final model. At stage $\delta$ with $\mathrm{cf} \delta=\omega_{1}$, suppose $\diamond$ gives us a $P_{\delta}$-name for a hereditarily separable and hereditarily Lindelöf space $\dot{X}$ with no countable network which is a subset of $R$ and is cometrizable with respect to the Euclidean topology. (Of course, we are using an appropriate coding of the diamond principle.)

If $\dot{X}$ contains an uncountable subspace $\dot{Z}$ whose topology is finer than the left or right Sorgenfrey topology, but there is no countable collection of closed sets satisfying the conditions of Lemma 2.1 with respect to $\dot{Z}$, then the proof of Proposition 2.6 shows that there is a ccc poset $Q_{\delta}^{1}$ which forces $Z$ to have an uncountable discrete subspace. (Note that $\mathrm{CH}$ holds in $V^{P_{\delta}}$.) If on the other hand $\dot{X}$ does not contain any subspace $Z$ whose topology is finer than the left or right Sorgenfrey topology, then the proof of the Main Result shows that there is a ccc poset $\dot{Q}_{\delta}^{2}$ which forces one. We let $\dot{Q}_{\delta}=\dot{Q}_{\delta}^{i}$, whichever applies; if neither applies, let $\dot{Q}_{\delta}=\{1\}$.

Now suppose that in $V^{P_{\omega_{2}}}$ we have a cometrizable counterexample $\dot{X}$ to the Main Result. We may assume that $\dot{X}$ is a subset of $R$ and is cometrizable with respect to the Euclidean topology. Since SOCA holds, we may also assume $\dot{X}$ is hereditarily separable and hereditarily Lindelöf (MA $\omega_{\omega_{1}}$ suffices-see footnote 6).

Let $\left\{\dot{x}_{\alpha}: \alpha<\theta\right\}$, where $\theta \in\left\{\omega_{1}, \omega_{2}\right\}$, be a one-to-one enumeration of $\dot{X}$, and let $\left\{\dot{F}_{\beta}^{\alpha}: \beta<\theta, \alpha<\lambda\right\}$, where $\lambda \in\left\{\omega_{1}, \omega_{2}\right\}$, enumerate closed subsets of $R$ such that $\left\{\dot{F}_{\beta}^{\alpha} \cap R: \alpha<\lambda\right\}$ is a neighborhood base at $\dot{x}_{\beta}$. Note that there are club many $\delta$ 's in $\omega_{2}$ of cofinality $\omega_{1}$ such that $\left\{\dot{x}_{\beta}: \beta<\theta \cap \delta\right\}$, $\left\{\dot{F}_{\beta}^{\alpha}: \beta<\theta \cap \delta, \alpha<\lambda \cap \delta\right\}$ is a $P_{\delta}$-name for a cometrizable space. Since $\mathrm{CH}$ holds and we are forcing with ccc posets of size $\aleph_{1}$, and since $\dot{X}$ has no countable network, there are club many $\delta<\omega_{2}$ of cofinality $\omega_{1}$ with the following property $N$ :

(N) For every $P_{\delta}$-name $\left\langle\dot{H}_{n}: n<\omega\right\rangle$ for a countable sequence of Euclidean closed sets, there exist $\beta \in \theta \cap \delta, \alpha \in \lambda \cap \delta$, and $\left\{\gamma_{n}: n<\omega\right\} \subset \theta \cap \delta$ such that $\dot{x}_{\beta}, \dot{x}_{\gamma_{n}}(n<\omega), \dot{F}_{\beta}^{\alpha}$ are all $P_{\delta}$-names and $P_{\delta}$ forces that $\dot{x}_{\gamma_{n}} \in \dot{H}_{n} \backslash \dot{F}_{\beta}^{\alpha}$ whenever $\dot{x}_{\beta} \in \dot{H}_{n}$.

Thus for club many $\delta$ 's, $\dot{X} \mid \delta$ is a $P_{\delta}$-name of a cometrizable space with no countable network; stationarily often, this will be what $\diamond$ gives us.

Now we see that $\dot{X}$ must contain an uncountable subspace $\dot{Z}$ with a topology finer than the left or right Sorgenfrey topology, for otherwise we would have forced this at some stage. Since $\mathrm{MA}_{\omega_{1}}$ holds in $V^{P_{\omega_{2}}}$, there is no countable collection $\mathscr{F}$ of Euclidean closed sets satisfying the conditions of Lemma 2.1 
with respect to $\dot{Z}$. As above, for club many $\delta<\omega_{2}$ of cofinality $\omega_{1}$ the same will be true for $\dot{Z} \mid \delta$. But then at some stage we will have forced $\dot{Z}$, and hence $\dot{X}$, to have an uncountable discrete subspace. This contradiction completes the proof.

One can also use the above technique to show that the consistency of the version of SOCA stated in footnote 5 follows from Con(ZFC).

\section{SOME REMARKS ON $(*)$}

In this section we make some further remarks concerning the consistency of the statement:

(*) Every uncountable first countable space contains an uncountable metrizable subspace or a copy of an uncountable subspace of the Sorgenfrey line.

Since a ZFC counterexample to $(*)$ is likely to be hereditarily separable and hereditarily Lindelöf (see the Introduction), one might begin by asking whether (*) holds for the class of cosmic spaces. Since no uncountable subspace of the Sorgenfrey line is cosmic, we are asking if every uncountable (first countable) cosmic space contains an uncountable metrizable subspace. The next result shows that this is consistent with and independent of ZFC.

Theorem 3.1. (a) ( $\left.\mathrm{MA}_{\omega_{1}}\right)$ Every uncountable cosmic space contains an uncountable metrizable subspace.

(b) $(\mathrm{CH})$ There is an uncountable first-countable cosmic space with no uncountable metrizable subspace.

Proof. (a) This is easy, and has been noticed before by Junilla and probably others. Let $(X, \tau)$ be a cosmic space of size $\aleph_{1}$. Let $\sigma_{1}$ be the separable metric topology on $X$ generated by a countable network. $X$ also has some weaker metric topology $\sigma_{2}$.

Let $f:\left(X, \sigma_{2}\right) \rightarrow\left(X, \sigma_{1}\right)$ be the identity on the set $X$. By $\left[\mathrm{Fr}_{1}\right.$, $23 M(\mathrm{f})]$, there is an uncountable $Y \subset X$ such that $f \mid Y$ is continuous. Then $\sigma_{1}, \sigma_{2}$, and $\tau$ agree on $Y$.

(b) This follows from the next two lemmas. Let $X$ be any first-countable nonmetrizable cosmic space of size $\aleph_{1}$. By Lemma 3.3, $X^{\omega}$ is not a countable union of metrizable subsets, so by Lemma 3.2, there is an uncountable $Y \subset X$ such that no uncountable subspace of $Y$ is metrizable.

Lemma 3.2. ( $\mathrm{CH})$ Suppose $X$ is hereditarily separable, of size and weight $\leq \aleph_{1}$, and suppose $X$ is not a countable union of metrizable subspaces. Then there is an uncountable $Y \subset X$ such that no uncountable subset of $Y$ is metrizable.

Proof. Let $\left\{X_{\alpha}: \alpha<\omega_{1}\right\}$ index all countable subsets of $X$, and let $\left\{B_{\alpha}: \alpha<\right.$ $\left.\omega_{1}\right\}$ be a base for $X$. Also let $\mathscr{B}_{\alpha}=\left\{B_{\beta}: \beta<\alpha\right\}$, and if $A \subset X$, let

$$
\mathscr{B}_{\alpha} \mid A=\left\{B \cap A: B \in \mathscr{B}_{\alpha}\right\} \text {. }
$$


For $\alpha<\omega_{1}$, inductively choose $x_{\alpha} \notin\left\{x_{\beta}: \beta<\alpha\right\}$ such that, if $\beta<\alpha$ and $x_{\alpha} \in \bar{X}_{\beta}$, then $\mathscr{B}_{\alpha} \mid X_{\beta} \cup\left\{x_{\alpha}\right\}$ does not contain a base for $x_{\alpha}$ in the subspace $X_{\beta} \cup\left\{x_{\alpha}\right\}$.

We claim that such an $x_{\alpha}$ always exists. If not, for each $x \in X \backslash\left\{x_{\beta}: \beta<\alpha\right\}$ let $\beta_{x}<\alpha$ be such that $x \in \bar{X}_{\beta_{x}}$ and $\mathscr{B}_{\alpha} \mid X_{\beta_{x}} \cup\{x\}$ contains a base for $x$ in $X_{\beta_{x}} \cup\{x\}$. Then $\left\{x: \beta_{x}=\beta\right\}$ is metrizable, so $X$ is a countable union of metrizable spaces, contrary to our hypothesis.

Let $Y=\left\{x_{\alpha}: \alpha<\omega_{1}\right\}$. Suppose $A \subset Y$ is uncountable and metrizable. Then $A$ is separable metric, so $\mathscr{B}_{\gamma} \mid A$ is a base for $A$ for some $\gamma<\omega_{1}$. Also, some $X_{\beta}$ is dense in $A$. Let $\alpha>\beta \cup \gamma$ such that $x_{\alpha} \in A$. Then $x_{\alpha} \in \bar{X}_{\beta}$ and $\mathscr{B}_{\alpha} \mid X_{\beta} \cup\left\{x_{\alpha}\right\}$ contains a base for $x_{\alpha}$, which contradicts the way $x_{\alpha}$ was chosen.

Lemma 3.3. If $X$ is not metrizable, then $X^{\omega}$ is not a countable union of separable metrizable subspaces.

Proof. Suppose $X$ is not a metrizable space, but $X^{\omega}=\bigcup_{n \in \omega} M_{n}$, where each $M_{n}$ is a separable metrizable space.

Let $\pi_{k}: X^{\omega} \rightarrow X^{k}$ be the projection. Choose, if possible, $n_{0} \in \omega$ and $\sigma_{0} \in$ $X^{n_{0}}$ such that $\sigma_{0} \notin \pi_{n_{0}}\left(M_{0}\right)$. Then choose, if possible, $n_{1}>n_{0}$ and $\sigma_{1} \in X^{n_{1}}$ such that $\sigma_{1} \mid n_{0}=\sigma_{0}$ and $\sigma_{1} \notin \pi_{n_{1}}\left(M_{1}\right)$. This cannot go on indefinitely, or we would have defined a point not in $\bigcup_{n \in \omega} M_{n}$. So there exists $n_{k} \in \omega$ and $\sigma_{k} \in X^{n_{k}}$ such that, if $n>n_{k}, \sigma \in X^{n}$, and $\sigma \mid n_{k}=\sigma_{k}$, then $\sigma \in \pi_{n}\left(M_{k}\right)$.

Let $Y=\left\{x \in M_{k}: x \mid n_{k}=\sigma_{k}\right\}$. If $\Pi: X^{\omega} \rightarrow X$ is the projection onto the $n_{k}$ th coordinate, then $\Pi \mid Y: Y \rightarrow X$ is open. $Y$ is separable metric, so $X$ is too, a contradiction.

As mentioned in the introduction, the consistency of $(*)$ would imply the consistency of

"Every uncountable first countable space contains an uncountable submetrizable subspace",

and we do not know if even this is consistent. However, we have the following result:

Theorem 3.4. $\left(\mathrm{MA}_{\omega_{1}}\right)$ If there is a counterexample to statement $(*)$, then there is a submetrizable one.

Proof. Suppose $(X, \tau)$ is a counterexample to $(*)$, with $|X|=\aleph_{1}$, and suppose also that $X$ contains no uncountable submetrizable subspace. We may assume $X \subset R$ (as a set). Let $\hat{\tau}$ be the topology on $X$ generated by $\tau$ together with the Euclidean topology.

We claim that $(X, \gamma)$ is also a counterexample. First of all, $(X, \gamma)$ contains no uncountable discrete subspaces, for otherwise $(X, \tau)$ would, too. Now suppose $(X, \hat{\tau})$ contains an uncountable (separable) metrizable subspace $X^{\prime}$. Then 
$\left(X^{\prime}, \tau\right)$ is cosmic, so contains an uncountable metrizable subspace by Lemma 3.1.

Finally, suppose $Y \subset X$ is uncountable, and $(Y, \hat{\tau})$ is homeomorphic to an uncountable subspace of the Sorgenfrey line. Applying $\left[\mathrm{Fr}_{1}, 23 M(\mathrm{f})\right]$ as in the proof of Lemma 3.1(a), we may assume that the separable metric topology on $Y$ with respect to which $(Y, \hat{\tau})$ is Sorgenfrey is the same as the Euclidean topology.

We aim for a contradiction by showing that $(Y, \tau)$ contains an uncountable submetrizable subspace. Let $\mathscr{B}$ be a countable base for $Y$ with the Euclidean topology $\mathscr{E}$. For $y \in Y$, let

$$
C_{y}=\left\{y^{\prime} \in Y: y \in \mathrm{cl}_{\tau} B \text { whenever } y^{\prime} \in B \in \mathscr{B}\right\} .
$$

Note that $C_{y}$ is Euclidean closed.

Claim. $C_{y}$ is countable.

If $\left|C_{y}\right|>\aleph_{0}$, let $y^{\prime} \in C_{y} \backslash\{y\}$ be a $\hat{\tau}$-limit point of $C_{y}$. Let $U$ be a $\hat{\tau}$ neighborhood of $y^{\prime}$ such that $U \backslash\left\{y^{\prime}\right\}$ is Euclidean open and $y \notin \mathrm{cl}_{\tau}(U)$. Pick $y^{\prime \prime} \in\left(U \backslash\left\{y^{\prime}\right\}\right) \cap C_{y}$. Then $y^{\prime \prime} \in B \subset U$ for some $B \in \mathscr{B}$; but $y \notin \mathrm{cl}_{\tau} B$, which contradicts $y^{\prime \prime} \in C_{y}$.

According to $\left[\mathrm{Fr}_{1}, 42 \mathrm{D}\right]$, under $\mathrm{MA}_{\omega_{1}}$ there exists an uncountable $Y^{\prime} \subset Y$ such that

$$
y_{1} \neq y_{2} \in Y^{\prime} \Rightarrow y_{1} \notin \mathrm{cl}_{\tau} B \text { for some } B \in \mathscr{B} \text { containing } Y_{2} .
$$

Now for $y \in Y^{\prime}$, let

$$
D_{y}=\left\{y^{\prime} \in Y^{\prime}: \mathrm{cl}_{\tau} B \cap \mathrm{cl}_{\tau} C \neq \varnothing \text { whenever } y \in B \in \mathscr{B} \text { and } y^{\prime} \in C \in \mathscr{B}\right\} \text {. }
$$

Claim. $\operatorname{cl}_{\mathscr{E}}\left(D_{y}\right)$ is countable.

If not, let $y^{\prime} \in \operatorname{cl}_{\mathscr{E}}\left(D_{y}\right)$ such that $y^{\prime} \neq y$ and $y^{\prime}$ is a $\hat{\tau}$-limit point of $\operatorname{cl}_{\mathscr{E}}\left(D_{y}\right)$. Let $y \in B \in \mathscr{B}$ such that $y^{\prime} \notin \mathrm{cl}_{\tau} B$. Choose a $\hat{\tau}$-neighborhood $U$ of $y^{\prime}$ such that $U \backslash\left\{y^{\prime}\right\}$ is Euclidean open and $\operatorname{cl}_{\tau} U \cap \mathrm{cl}_{\tau} B=\varnothing$ (use the regularity of $\tau$ here). Since $\left(U \backslash\left\{y^{\prime}\right\}\right) \cap \operatorname{cl}_{\mathscr{E}}\left(D_{y}\right) \neq \varnothing$, we can pick $y^{\prime \prime} \in\left(U \backslash\left\{y^{\prime}\right\}\right) \cap D_{y}$. Then $y^{\prime \prime} \in C \subset U$ for some $C \in \mathscr{B}$, and $\operatorname{cl}_{\tau} C \cap \mathrm{cl}_{\tau} B=\varnothing$, a contradiction.

By the claims, assuming $M A_{\omega_{1}}$ we can pass to an uncountable $X^{\prime \prime} \subset Y^{\prime}$ such that distinct points of $X^{\prime \prime}$ have Euclidean neighborhoods with disjoint $\tau$-closures. We may assume that $X^{\prime \prime}=\left\{x_{\alpha}: \alpha<\omega_{1}\right\}$, that $\left|B \cap X^{\prime \prime}\right|=\aleph_{1}$ for each $B \in \mathscr{B}$, and that $\mathscr{B}$ is closed under finite unions.

Let $(P, \leq)$ be the poset consisting of all pairs $(\mathscr{F}, F)$, where $\mathscr{F} \in[\mathscr{B}]^{<\aleph_{0}}$ and $F \in\left[X^{\prime \prime}\right]^{<\aleph_{0}}$ satisfying:

(i) $(F, \supset)$ is isomorphic to an initial part of the Cantor tree ${ }^{<\omega} 2$ of the form ${ }^{<n} 2$, for some $n \in \omega$;

(ii) If $B, B^{\prime} \in \mathscr{F}$ are on the same level, then $\operatorname{cl}_{\tau} B \cap \mathrm{cl}_{\tau} B^{\prime}=\varnothing$;

(iii) Each $x \in F$ is in the intersection of some branch of $(\mathscr{F}, \supset)$. 
We define $\left(\mathscr{F}^{\prime}, F^{\prime}\right) \leq(\mathscr{F}, F)$ iff $\mathscr{F}^{\prime} \supset \mathscr{F}$ and $F^{\prime} \supset F$.

Since $[\mathscr{B}]^{<\aleph_{0}}$ is countable, it is clear that $(P, \leq)$ is ccc.

For $\alpha<\omega_{1}$, let

$$
D_{\alpha}=\left\{(\mathscr{F}, F) \in P: x_{\beta} \in F \text { for some } \beta>\alpha\right\},
$$

and for $x \in X^{\prime \prime}$, let

$$
E_{x}=\{(\mathscr{F}, F): x \notin F \text { or } B \cap F=\{x\} \text { for some } B \in \mathscr{F}\} \text {. }
$$

Clearly the $D_{\alpha}$ 's and $E_{x}$ 's are dense. Let $\mathscr{G}$ be a filter meeting them all, let

$$
Z=\bigcup\{F:(\mathscr{F}, F) \in \mathscr{G} \text { for some } \mathscr{F} \subset \mathscr{B}\},
$$

and let

$$
\mathscr{C}=\bigcup\left\{\mathscr{F}:(\mathscr{F}, F) \in \mathscr{F} \text { for some } F \subset X^{\prime \prime}\right\}
$$

Then $(\mathscr{C}, \supset)$ is isomorphic to a Cantor tree, and each $y \in Y$ is in the intersection of some branch. Since $\mathscr{G}$ meets each $E_{x}$, the intersection of each branch contains at most one point of $Z$. By property (ii), each $C \cap Z$ for $C \in \mathscr{C}$ is $\tau$-open and Euclidean closed. Hence $\{C \cap Z: C \in \mathscr{C}\}$ is a base for a metrizable topology on $Z$ with respect to which $(Z, \tau \mid Z)$ is cometrizable.

\section{REFERENCES}

[A] A. V. Arhangel' skii, The structure and classification of topological spaces and cardinal invariants, Russian Math. Surveys 33 (1978), 33-96.

[ARS] U. Abraham, M. Rubin, and S. Shelah, On the consistency of some partition theorems for continuous colorings, and the structure of $\aleph_{1}$-dense real order types, Ann. Pure Appl. Logic 29 (1985), 123-206.

[AS] U. Abraham and S. Shelah, Martin's Axiom does not imply that every two $\aleph_{1}$-dense sets of reals are isomorphic, Israel J. Math. 38 (1981), 161-176.

[ $\mathrm{B}_{1}$ ] J. Baumgartner, Applications of the proper forcing axiom, Handbook of Set-Theoretic Topology, edited by K. Kunen and J. E. Vaughan, North-Holland, Amsterdam, 1984, pp. 914-959.

[ $\mathrm{B}_{2}$ ] _ All $\aleph_{1}$-dense sets of reals can be isomorphic, Fund. Math. 79 (1973), 101-106.

[Br] E. S. Berney, A regular Lindelöf semi-metric space which has no countable network, Proc. Amer. Math. Soc. 26 (1970), 361-364.

[Bs] A. Beslagic, Embedding cosmic spaces in Lusin spaces, Proc. Amer. Math. Soc. 89 (1983), 515-518.

[BvD] D. K. Burke and E. K. van Douwen, No dense metrizable $G_{\delta}$-subspaces in butterfly semimetrizable spaces, Topology Appl. 11 (1980), 31-36.

[C] K. Ciesielski, Martin's Axiom and a regular topological space with uncountable netweight whose countable product is hereditarily separable and hereditarily Lindelöf, J. Symbolic Logic 52 (1987), 396-399.

[vDK] E. van Douwen and K. Kunen, L-spaces and S-spaces in $P(\omega)$, Topology Appl. 14 (1982), 143-149.

[ $\mathrm{Fr}_{1}$ ] D. H. Fremlin, Consequences of Martin's Axiom, Cambridge Univ. Press, 1984.

[ $\left.\mathrm{Fr}_{2}\right]$, Notes on Martin's maximum, unpublished notes.

[G] G. Gruenhage, On the existence of metrizable or Sorgenfrey subspaces, General Topology and its Relation to Modern Algebra and Analysis (Proc. Sixth Prague Topology Sympos., 1986, (Z. Frolik, ed.), Heldermann-Verlag, Berlin, 1986, pp. 223-230. 
[H] R. W. Heath, On certain first-countable spaces, Topology Seminar (Wis., 1965), Ann. of Math. Studies, no 60, Princeton Univ. Press, Princeton, N.J., 1966, pp. 103-113.

[J] I. Juhasz, SETOP Conference notes, Toronto, Canada, 1980.

[JKR] I. Juhasz, K. Kunen, and M. E. Rudin, Two more hereditarily separable non-Lindelöf spaces, Canad. J. Math. 28 (1976), 998-1005.

[M] E. A. Michael, Paracompactness and the Lindelöf property in finite and countable Cartesian products, Comput. Math. 23 (1971), 199-214.

[S] S. Shelah, Proper forcing, Lecture Notes in Math., vol. 940, Springer-Verlag, Berlin, 1982.

[Sz] Z. Szentmiklossy, S-spaces and L-spaces under Martin's Axiom, Colloq. Math. Soc. János Bolyai 23 (Budapest 1978 (II)), North-Holland, Amsterdam, 1980, pp. 1139-1145.

[Ta] F. Tall, The density topology, Pacific J. Math. 75 (1976), 275-284.

[Tk] M. G. Tkačenko, Chains and cardinals, Dokl. Akad. Nauk SSSR 239 (1978), 546-549.

[ $\mathrm{To}_{1}$ ] S. Todorcevic, Forcing positive partition relations, Trans. Amer. Math. Soc. 280 (1983), 703-720.

[ $\left.\mathrm{To}_{2}\right]$ _ Remarks on cellularity in products, Compositio Math. 57 (1986), 357-372.

[ $\left.\mathrm{To}_{3}\right]$, A class of spaces associated with gaps, unpublished manuscript.

[V] N. V. Veličko, Symmetrizable spaces, Mat. Zametki 12 (1972), 577-582=Math. Notes 12 (1972), 784-786.

Mathematics-Foundations, Analysis, And Topology, Auburn University, Auburn, AlABAMA 36849 